\title{
ON THE DOUBLE COMMUTATOR ALGEBRA OF QF-3 ALGEBRAS
}

\author{
H. Y. MOCHIZUKI
}

1. Introduction and Preliminaries. Nakayama [9] suggested that algebras be classified according to the length of a right projective, injective resolution of the algebra as a left module. Tachikawa [10] showed that an algebra is QF. 3 (see [12] for definition) if and only if the length is at least one. He [11] also gave some estimations of the length of the resolution besides relating the length to the size of the double commutator algebra of a faithful projective, injective left ideal.

All algebras considered here are finite-dimensional algebras over a fixed field $k$. All modules are unital and finitely generated. Let $B$ be an algebra with Jacobson radical $N$. Left $B$-module $M$ is called an essential extension of submodule $K$ if for each submodule $L$ of $M$ such that $L \neq(0), K \cap L \neq(0)$. For algebras this definition is equivalent to the condition that the left socles $l(M)$ and $l(K)$ of $M$ and $K$ coincide (the left socle $l(X)$ of $B$-module $X$ is the semi-simple part of $X$, i.e. $l(X)=\{x \in X: N x=0\})$.

Eckmann and Schopf [4] have shown that every $B$-module $M$ is a submodule of a unique minimal injective B-module $E(M)$, called the minimal injective of $M$ and characterized by that the property that it is the "largest" essential extension of $M . E(M)$ is the minimal injective of $M$ if and only if it is the minimal injective of $l(M)$.

Nagao and Nakayama [8, Theorem 2, p. 169] showed that any indecomposable injective left $B$-module is the vector space dual of a primitive right ideal of $B$ (i.e. an indecomposable projective right $B$-module) and is therefore a finite-dimensional vector space over $k$. Since the indecomposable injectives are just the minimal injectives of simple $B$-modules [7, Theorem 3.1, p. 221] and the left socle of any (finitely generated) left $B$-module $K$ is the direct sum of a finite number of simple $B$-modules, the minimal injective of $K$ is the direct 
sum of a finite number of indecomposable injectives and is therefore finitedimensional over $k$.

An injective resolution of $K$

$$
0 \rightarrow K \longrightarrow X_{1} \stackrel{\delta_{1}}{\rightarrow} X_{2} \stackrel{\delta_{2}}{\rightarrow} \cdots \longrightarrow X_{n}
$$

is called minimal if $X_{i}$ is the minimal injective of $\operatorname{Im} \delta_{i}-1$. If (1) is a minimal injective resolution such that each $X_{i}$ is also projective, then we say, after Tachikawa [11], that $K$ has dominant dimension $\geqq n$, where the zero module is considered to be projective and injective (dominant dimension of $K$ is denoted by domi. $\operatorname{dim}_{B} K$ ). If $X_{1}$ is not projective, then $\operatorname{dimi} \cdot \operatorname{dim}_{B} K=0$; if the largest such integer is $m$, then domi. $\operatorname{dim}_{B} K=m$; if no largest such integer exists, then domi. $\operatorname{dim}_{B} K=\infty$. As we mentioned before Tachikawa proved that $B$ is $Q F-3$ if and only if domi. $\operatorname{dim}_{B} B \geqq 1$ (regardless of which side $B$ operates on itself). Therefore we are restricted to $Q F-3$ algebras.

Suppose then that $B$ is $Q F-3$. There is an idempotent $e$ of $B$ such that $B e$ is a faithful projective, injective left ideal of $B$. Considering the $B$-endomorphism algebra $A$ of $B e$, i.e. the commutator algebra of $B e$, as a right operator domain, we have that $B e$ is a $(B, A)$-module (we consider only bimodules which are two-sded and on both sides of which elements of $k$ operate the same way). We shall identify $A$ with $e B e$. Then, according to a result of Morita [6, Theorem 17.5, p. 137], the $A$-endomorphism algebra $B^{\prime}$ of $B e$ is a $Q F-3$ algebra, and $B$ is a subalgebra of $B^{\prime}$ such that $B$ contains the identity of $B^{\prime}$ and $B e=B^{\prime} e$.

In $\S 2$ we show that for hereditary $Q F-3$ algebra $B$ (see pp. 12-14 of [3] for definition and properties of hereditary algebras), $A$ and therefore $B^{\prime}$ are semi-simple algebras. Furthermore $B^{\prime}$ is the minimal injective of $B$ both as left and right $B$-modules.

In $\S 3$ we prove that if $B$ is a $Q F-3$ algebra with domi. $\operatorname{dim}_{B} B=1$, then $B^{\prime}$ is strictly larger than $B$. This theorem is the converse of Theorem 1.4 of [11].

2. Hereditary algebras. Let $B$ denote a hereditary $Q F-3$ algebra with faithful projective, injective left ideal $B e, e$ an idempotent. If $P$ is any projective left $B$-module, then $P$ can be embedded in a direct sum of copies of $B e$. The minimal injective $E(P)$ of $P$, being a direct summand of this direct sum, 
must be projective.

Lemma 2.1. In the above setting, $\operatorname{Hom}_{B}(E(P) / P, E(P))=(0)$.

Proof. Suppose there exists homomorphism $f \neq 0$ in $\operatorname{Hom}_{B}(E(P) / P, E(P))$. Then $f$ induces a $B$-homomorphism $g$ from $E(P)$ into $E(P)$ such that $P^{\prime}=$ $g(E(P)) \neq(0)$ and the kernel, Ker $g$, of $g$ contains $P$. We therefore have an exact sequence.

$$
(0) \rightarrow \operatorname{Ker} g \rightarrow E(P) \rightarrow P^{\prime} \rightarrow(0),
$$

where $P^{\prime}$ is projective, being a submodule of $E(P)$. (2.1) must split, implying that $P$ is contained in a direct summand of $E(P)$. But then $E(P)$ cannot be an essential extension of $P$. We must conclude that $\operatorname{Hom}_{B}(E(P) / P, E(P))=(0)$.

Q.E.D.

Leмma 2.2. If $E$ is a projective, injective left $B$-module, then $\operatorname{Hom}_{B}(E, E)$ is a semi-simple algebra.

Proof. The left socle $l(E)$ of $E$ is projective, and $E$ is the minimal injective of $l(E)$. We therefore have the exact sequence

$$
(0) \longrightarrow l(E) \stackrel{j}{\longrightarrow} E \longrightarrow E / l(E) \longrightarrow(0),
$$

After "Homming" into $E$ we get the exact sequence

$$
\operatorname{Hom}_{B}(E / l(E), E)=(0) \longrightarrow \operatorname{Hom}_{B}(E, E) \stackrel{j^{*}}{\rightarrow} \operatorname{Hom}_{B}(l(E), E) \longrightarrow(0) .
$$

For each $f \in \operatorname{Hom}_{B}(l(E), E), f(l(E)) \subseteq l(E)$. Thus as abelian groups we have isomorphism

$$
k: \operatorname{Hom}_{B}(l(E), E) \approx \operatorname{Hom}_{B}(l(E), l(E)) .
$$

We make the assertion that the map

$$
\boldsymbol{k} \circ j^{*}: \operatorname{Hom}_{B}(E, E) \longrightarrow \operatorname{Hom}_{B}(l(E), l(E))
$$

is an isomorphism of algebras. $k \circ j^{*}$ is clearly an isomorphism of abelian groups. For $f, g \in \operatorname{Hom}_{B}(E, E)$ and for $x \in l(E)$,

$$
\begin{aligned}
k \circ j^{*}(g \circ f)(x) & =g \circ f(x) \\
& =g\left[k \circ j^{*}(f)(x)\right] \\
& =\left[k \circ j^{*}(g)\right] \circ\left[k \circ j^{*}(f)\right](x)
\end{aligned}
$$

i.e. $k \circ j^{*}(g \circ f)=\left[k \circ j^{*}(g)\right] \circ\left[k \circ j^{*}(f)\right]$ 
It is also clear that $k^{\circ} j^{*}(\alpha)=\alpha$ for all elements $\alpha$ in $k$. Thus our assertion is proved.

$l(E)$ is the direct sum of a finite number of simple $B$-modules. Therefore $\operatorname{Hom}_{B}(l(E), l(E))$ is a semi-simple algebra $[2, \S 5$, Exercise 17, p. 61]. The isomorphism (2.2) then gives us the lemma.

Q.E.D.

We are now ready to state the main theorem of this section.

THEOREM 2.3. Let $B$ be a finite dimensional hereditary algebra over field $k$ and let $e$ be an idempotent such that $B e$ is a faithful projective, injective left ideal of $B$. Then the following facts hold.

(1) Both the commutator algebra $A=e B e$ and the double commutator algebra $B^{\prime}$ are semi-simple.

(2) $B^{\prime}$ is $a(B, B)$-module which is both the left and right minimal injective of $B$.

(3) $B$ is a generalized uniserial algebra, i.e. every indecomposable left or right projective $B$-module has a unique composition series.

Proof. That $A$ is semi simple is a result of Lemma 2.2. On the other hand $B^{\prime}$ is semi-simple by $[2, \S 5$, Exer. 17, p. 61$]$ since $B e$ is the direct sum of finitely many right simple $A$-modules. Therefore we have proved (1).

We complete the proof of Theorem 2.3 by a series of lemmas. Recall that $B$ is a subalgebra of $B^{\prime}$ such that the identities of $B$ and $B^{\prime}$ coincide and $B e$ $=B^{\prime} e$. Let $e^{\prime}=1-e$, an idempotent orthogonal to $e$. Since $B^{\prime}$ is semi-simple we know that $B^{\prime}=B^{\prime} e \oplus B^{\prime} e^{\prime}$ where

(a) $B^{\prime} e=\oplus \sum B^{\prime} e_{i}(1 \leqq i \leqq m)$ and

$$
B^{\prime} e^{\prime}=\oplus \sum B^{\prime} e_{j}^{\prime}(1 \leqq j \leqq n)
$$

(b) the $e_{i}$ and $e_{j}^{\prime}$ are mutually orthogonal primitive idempotents in $B^{\prime}$.

(c) each $B^{\prime} e_{i}$ and each $B^{\prime} e_{j}^{\prime}$ is a minimal left ideal of $B^{\prime}$.

Lemma 2.4. $B^{\prime}$ is a projective, injective left $B$-module.

Proof. Since $B^{\prime}=B^{\prime} e \oplus B^{\prime} e^{\prime}$ and $B^{\prime}$ is a projective and injective left $B$ module, we must show that $B^{\prime} e^{\prime}$ is a projective, injective left $B$-module. Every minimal right ideal of $A$ is isomorphic to a direct summand of the right $A$ module $B \boldsymbol{e}$ [6, Theorem 17.5]. This fact together with the semi-simplicity of $A$ implies that $B e$ is a faithful right $A$-module. Therefore, by Theorem 17.2 of [6], $B e=B^{\prime} e$ is a faithful left $B^{\prime}$-module. But then this fact means that 
each $B^{\prime} e_{j}^{\prime}$ is isomorphic to a direct summand of $B^{\prime} e$ as left $B^{\prime}$-modules and therefore as left $B$-modules. We conclude that $B^{\prime} e^{\prime}$ is a projective, injective left $B$-module.

Q.E.D.

Continuing our discussion, we note that $B^{\prime}$, being semi-simple, is a Frobenius algebra. Therefore, as a right $B^{\prime}$-module, $B^{\prime} \approx \operatorname{Hom}_{k}\left(B^{\prime}, k\right)$. This isomorphism is also a $B$-isomorphism under the restriction of the algebra of operators, implying that $B^{\prime}$ is also a projective, injective right $B^{\prime}$ module containing $B$ as a submodule. Furthermore, by Theorem 17.5 of [6] there is idempotent $f$ of $B^{\prime}$ such that $f B=f B^{\prime} \subseteq B$ and $f B^{\prime}$ is a faithful right $B^{\prime}$ module. We shall prove that $B^{\prime}$ is the left minimal injective of $B$. That $B^{\prime}$ is the right minimal injective of $B$ will follow similarly.

We need the following lemma, due to Tachikawa [11], the proof of which we repeat here.

Lemma 2.5. If $P$ is a projective, injective left $B$-module, then $B^{\prime} \otimes{ }_{B} P \approx P$ as left $B$-modules.

Proof. It is clear from the additivity of the functor $B^{\prime} \otimes_{B}$ that we need only prove this lemma for $P$ indecomposable. Then $P$ is isomorphic to $B e_{i}=$ $B^{\prime} e_{i}$ for some $i$. We may assume $P=B e_{i}$.

$\otimes_{B} B e_{i}$ is an exact functor on the category of right $B$-modules. If we apply this functor to the exact sequence of two-sided $(B, B)$-modules

$$
(0) \longrightarrow B \stackrel{\delta}{\rightarrow} B^{\prime},
$$

we get the exact sequence of left $B$-modules

$$
(0) \longrightarrow B \otimes{ }_{B} B e_{i} \stackrel{\delta \otimes 1}{\longrightarrow} B^{\prime} \otimes{ }_{B} B e_{i} .
$$

As left $B$-modules $B \otimes_{B} B e_{i} \approx B e_{i}$.

We shall show that $\delta \otimes 1$ is an isomorphism by showing that $\delta \otimes 1$ is an epimorphism. We regard $B \otimes{ }_{B} B e_{i}$ as a submodule of $B^{\prime} \otimes_{B} B e_{i}$. Let $b^{\prime} \otimes b e_{i}$ be a generator of $B^{\prime} \otimes{ }_{B} B e_{i}$. Then $b^{\prime} \otimes b e_{i}=b^{\prime} b e_{i} \otimes e_{i}$. But since $B^{\prime} e_{i}=B e_{i}$, $b^{\prime} b e_{i} \in B e_{i}$, and therefore $b^{\prime} \otimes b e^{\prime}=1 \otimes b^{\prime} b e_{i} \in B \otimes{ }_{B} B e_{i}$. It follows that $B e_{i} \approx B^{\prime}$ $\otimes{ }_{B} B e_{i}$.

Q.E.D.

Since $B^{\prime}$ is an injective left $B$-module containing $B, B^{\prime}$ also contains the minimal injective $E(B)$ of $B$. Thus we have the exact sequences of $B$-modules $(0) \rightarrow B \rightarrow E(B)$ and $(0) \rightarrow E(B) \rightarrow B^{\prime}$. Applying the exact functor $B^{\prime} \otimes_{B}$ to these 
sequences we get the exact sequences of $B$-modules $(0) \rightarrow B^{\prime} \otimes_{B} B \rightarrow B^{\prime} \otimes_{B} E(B)$ and $(0) \rightarrow B^{\prime} \otimes{ }_{B} E(B) \rightarrow B^{\prime} \otimes{ }_{B} B^{\prime}$.

As left $B$-modules $B^{\prime} \otimes_{B} B \approx B^{\prime} . \quad$ By Lemma $2.5 B^{\prime} \otimes{ }_{B} E(B)$ and $B^{\prime} \otimes_{B} B^{\prime}$ are isomorphic to $E(B)$ and to $B^{\prime}$ respectively since the latter two are also projective. Combining these results together with a vector space dimension argument, we see that $E(B)=B^{\prime}$. We have now completed the proof of (2).

To prove (3) we need two more lemmas.

Lемма 2.6. If $E$ is an indecomposable projective, injective left $B$-module, then $E$ is uniserial, i.e. E has a unique composition series.

Proof. Let $N$ denote the Jacobson radical of $B$. $E$, being both projective and injective, has a unique maximal submodule and unique minimal submodule, namely $N E$ and the socle $l(E)$ of $E$ respectively. Recalling that $B$ is hereditary, we note that $N E$, being a submodule of a projective, is also proiective. Furthermore, $N E$ has the same unique minimal submodule as $E$ and is therefore indecomposable. $N E$ has a unique maximal submodule $N^{2} E$.

If we continue in this manner, we find that for each $i$ for which $N^{i} E \neq(0)$, $N^{i} E=l(E)$ or $N^{i} E$ has a unique maximal submodule $N^{i+1} E \neq(0)$. Let $(0)=E_{0}$ $\subset E_{1} \subset E_{2} \subset \cdots \subset E_{m}=E$ be a composition series for $E$. By what we have shown above we have that $N^{i} E=E_{m-i}, i=0,1, \ldots, m$ where $N^{o}=B$ and $N^{m} E=(0)$. It follows that $E$ has a unique composition series and is uniserial.

Q.E.D.

LемMA 2.7. The minimal injective $E(P)$ of an indecomposable projective left $B$-module $P$ is indecomposable.

Proof. Suppose $E(P)=E_{1} \oplus E_{2}$ where $E_{1}$ and $E_{2}$ are nontrivial submodules of $E(P)$. Let $\pi$ be the projection map of $P$ into $E_{1}$. Since $B$ is hereditary and $E(P)$ is an essential extension of $P, \pi(P) \neq 0$, and therefore $\pi(P)$ is projective. This fact implies that $\pi(P)$ is isomorphic to a direct summand of $P$, and it follows from the indecomposability of $P$ that $\pi(P)$ is isomorphic to $P$. But then $E_{1}$ is an injective left $B$-module which contains a copy of $P$ and the dimension of $E_{1}$ over $k$ is less than the dimension of $E(P)$ over $k$. Having contradicted the choice of $E(P)$, we conclude that $E(P)$ is indecomposable.

Q.E.D.

Remark. Lemmas 2.6 and 2.7 also hold when right is substituted for left. 
In fact, the proofs are exactly the same except for the substitution of right for left since the property of being hereditary is two-sided for $B$.

(3) can be proved very easily. Let $P$ be any left or right indecomposable projective $B$-module. Then its minimal injective, being indecomposable by Lemma 2.7 and the above remark, has a unique composition series by Lemma 2.6. Since a composition series for $P$ must be part of some composition series for $E(P)$, it follows that $P$ also has a unique composition series. $B$ is therefore a generalized uniserial algebra. The proof of Theorem 2.3 is now completed.

Remarks. Let $B$ be a hereditary $Q F-3$ algebra with faithful projective, injective left ideal $B e, e$ an idempotent in $B$, and $B^{\prime}$ its double commutator. Let $1=\sum e_{i}(1 \leqq i \leqq n)$ where the $e_{i}$ are mutually orthogonal primitive idempotents in $B$. We assert that the $e_{i}$ are also mutually orthogonal primitive idempotents in $B^{\prime}$.

For each $i, B e_{i}$ is an indecomposable projective left $B$-module. Furthermore, $B e_{i}$ is a submodule of the projective, injective left $B$-module $B^{\prime} e_{i}$. By Theorem $2.3 B^{\prime}=\oplus \sum B^{\prime} e_{i}(1 \leqq i \leqq n)$ is the minimal injective of $B$, and it is clear that $B^{\prime}$ the minimal injective of $B$ implies that $B^{\prime} \boldsymbol{e}_{i}$ is the minimal injective of $B \boldsymbol{e}_{i}$. Hence, Lemma 2.7 tells us that $B^{\prime} e_{i}$ is indecomposable as a left $B$-module and as a left $B^{\prime}$-module. This latter fact implies that $\boldsymbol{e}_{i}$ is a primitive idempotent in $B^{\prime}$.

Theorem 2.3 and these remarks tell us that $B$ is a direct sum of ideals, one for each (unique) simple ideal direct summand of $B^{\prime}$ in which it is contained. Furthermore, a canonical example of such an ideal direct summand of $B$ is the set of all (lower) triangular matrices over a semi-simple ring with minimum conditions.

3. Dominant dimension of the algebra equals 1 . Let $B$ be a $Q F-3$ algebra. Thrall [12, Theorem 5, p. 179] proved that the unique minimal faithful left $B$ module $Q$ is a direct sum of one copy each of the dominant primitive left ideals of $B$. A primitive left ideal is called dominant if it is a dual as a vector space over $k$ to a primitive right ideal $[12, \mathrm{p} .174]$. They are thus injective [cf. §1]. We let $e$ be an idempotent of $B$ such that $B e$ is a faithful projective, injective left ideal of $B$.

We recall these facts from $\S 1$. We know that the $B$-endomorphism algebra 
of $B e$ is $A=e B e$ [5, Chapter III, Proposition 7, p. 51], with the elements of $A$ acting on $B e$ by right multiplication. If $B^{\prime}$ denotes the $A$-endomorphism algebra of $B e$ as left operator domain, then $B$ is a subalgebra of $B^{\prime}$, the identity 1 of $B^{\prime}$ is in $B$, and $B e=B^{\prime} e$. Thus $B^{\prime}$ acts on $B e$ by left multiplication as elements of $B^{\prime}$. In the usual language, $B^{\prime}$ is called the double centralizer or double commutator algebra of left $B$-module $B e$.

ThEOREM 3.1. Let $B$ be a QF-3 algebra, and let $e$ be an idempotent of $B$ such that $B e$ is a faithful projective, injective left $B$-module. If $\operatorname{domi}_{\text {. }} \operatorname{dim}_{B} B=1$, then $B$ is a proper subalgebra of $B^{\prime}$, the double commutator algebra of left $B$ module $\mathrm{Be}$.

Remark. This theorem is the converse of Theorem 1.4 of [11], which states that if domi. $\operatorname{dim}_{B} B>1$, then $B=B^{\prime}$.

We prove theorem by a series of Lemmas.

As we have seen in $\S 2$, the minimal injective $E(B)$ of $B$ is projective. Therefore $E(B)$ is a direct sum of copies of dominant left ideals. Letting $\boldsymbol{e}^{\prime}$ $=1-e$, we have the Peirce decomposition of $B$ into left ideals, $B=B e \oplus B e^{\prime}$. If $E$ is the minimal injective of $B \varepsilon^{\prime}$, then clearly $E(B)=B e \oplus E$. $E$, being both projective and injective, is the direct sum of finitely many dominant primitive left ideals. We therefore get exact sequence

$$
(0) \longrightarrow B \longrightarrow B e \oplus E \longrightarrow E / B e^{\prime} \longrightarrow(0) \text {. }
$$

Thus domi. $\operatorname{dim}_{B} B=$ domi. $\operatorname{dim}_{B} E / B e^{\prime}-1=$ domi. $\operatorname{dim}_{B} B e^{\prime}$.

Suppose domi. $\operatorname{dim}_{B} B=1$. Then we have exact sequence.

$$
(0) \longrightarrow B e^{\prime} \longrightarrow E \stackrel{\text { II }}{\longrightarrow} E / B e^{\prime} \longrightarrow(0)
$$

where $E / B e^{\prime}$ cannot be embedded in any projective, injective left $B$-module. Clearly, $E / B e^{\prime}$ cannot be embedded in any projective $B$-module. Since $E / B e^{\prime}$ is finitely generated, 4.2 and 4.5 of $[1$, p. 477$]$ implies that the intersection $K$ in $E / B e^{\prime}$ of all the kernels of maps in $\left(E / B e^{\prime}\right)^{\#}$ is a nontrivial left $B$-module. (If $X$ is a left $B$-module, we let $X^{\#}=\operatorname{Hom}_{B}(X, B e)$ ).

Lemma 3.2. $K^{\#}=(0)$.

Proof. Any $B$-homomorphism of $K$ into $B e$ can be extended to a $B$-homomorphism of $E / B e^{\prime}$ into $B e$ since $B e$ is injective. Thus by the definition of 
$K$, the homomorphism must be trivial on $K$.

Q.E.D.

We let $M$ denote $\Pi^{-1}(K)$, the submodule of $E$ corresponding to $K$. From the exact sequence

$$
(0) \longrightarrow B e^{\prime} \longrightarrow M \longrightarrow M / B e^{\prime}=K \longrightarrow(0)
$$

we get the exact sequence

$$
(0) \longrightarrow K^{\#}=(0) \longrightarrow M^{\#} \longrightarrow\left(B e^{\prime}\right)^{\#} \longrightarrow(0)
$$

where exactness on the right follows from the injectivness of $B e$. We see that $M^{\#}$ and $\left(B e^{\prime}\right)^{\#}$ are isomorphic with respect to the dual map of the embedding maps of $B e^{\prime}$ in $M$. Thus every $B$-homomorphism of $B e^{\prime}$ into $B e$ extends uniquely to a $B$-homomorphism of $M$ into $B e$.

Since $B$ is a subalgebra of $B^{\prime}$, the inclusion of $B e^{\prime}$ in $B^{\prime}$ is actually a left $B$-monomorphism. Thus for each $b \in B e$, the map $x \rightarrow x b, x \in B \boldsymbol{e}^{\prime}$ is a $B$-homomorphism of $B e^{\prime}$ into $B e$. We can extend this map uniquely to a $B$-homomorphism of $M$ into $B e$. We shall also denote this map by $x \rightarrow x b, x \in M$. Thus $x_{1}+x_{2} \rightarrow x_{1} b+x_{2} b, x_{1}, x_{2} \in M$, and $(r x) b=r(x b), r \in B, x \in M$.

Lemma 3.3. For each $x \in M$, the map $b \rightarrow x b, b \in B e$, is an A-endomorphism of Be. Furthermore, $x \in M$ induces the trivial endomorphism if and only if $x=$ 0 .

Proof. That $b_{1}+b_{2} \rightarrow x b_{1}+x b_{2}$ is clear. Let $c \in A$. Since for $b \in B e^{\prime}$, multiplication of $b$ and $c$ as elements of $B$ represents functional composition when considered as homomorphisms, we have $x(b c)=(x b) c$. Thus the first half of our assertion is proved.

Let $M_{1}=\{x \in M: x b=0$ for all $b \in B e\}$. Clearly, $M_{1}$ is a $B$-submodule of $M$. Since $M$ is an essential extension of $B e^{\prime}, M_{1} \cap B e^{\prime} \neq(0)$ if $M_{1} \neq(0)$. But since $y B e \neq(0)$ for all nonzero elements $y$ in $B e^{\prime}$ (recall that $B e$ is faithful), $M_{1}=(0)$.

Q.E.D.

We define a map of $M$ into $B^{\prime}$ by $x \rightarrow r^{\prime}$ if $x b=r^{\prime} b$ for all $b \in B e$. By Lemma 4.3 and the definition of $B^{\prime}$, this definition makes sense. Since $B^{\prime}$ acts faithfully on $B e$, this map is the inclusion map on $B e^{\prime}$. By the remarks preceding Lemma 3.3 together with Lemma 3.3 , this map is actually a $B$-monomorphism of $M$ into $B^{\prime}$. We now identify $M$ with its image in $B^{\prime}$, which is a $B$-submodule of $B^{\prime}$ which properly contains $B e^{\prime}$ as a $B$-submodule. 
LeMma 3.4. $M \cap B^{\prime} e=(0)$.

Proof. Suppose $M \cap B^{\prime} e=M_{2} \neq(0)$. Since $M_{2}$ is a left $B$-module, $M_{2} \cap B e^{\prime}$ $\neq(0)$ (we recall that $M$ is an essential extension of $B e^{\prime}$ ). But $B e=B^{\prime} e$, implying that $(0)=B e \cap B e^{\prime} \supseteq M_{2} \cap B e^{\prime} \neq(0)$. Since our supposition leads to a contradiction, we must conclude that $M \cap B^{\prime} e=(0)$.

Q.E.D.

We now have the inclusions $B^{\prime} \supseteq B e \oplus M \supset B e \oplus B e^{\prime}=B$ where the last inclusion is proper. We have therefore proved Theorem 4.1.

\section{REFERENCES}

[1] H. Bass, Finitistic dimension and a homological generalization of semi-primary rings, Trans. Amer. Math. Soc. 95 (1960), 446-488.

[2] N. Bourbaki, Algèbre. Chap. VIII, "Modules et anneaux semi-simples," Hermann, Paris, 1958.

[ 3 ] H. Cartan and S. Eilenberg, Homological algebra, Princeton University Press, 1956.

[ 4 ] B. Eckmann and A. Schopf, Über injecktive Moduln, Archiv der Math. 4 (1953), 75-78.

[5] N. Jacobson, The structure of rings, Am. Math. Soc., Providence, 1956.

[6] K. Morita, Duality for modules and its applications to the theory of rings with minimum condition, Sci. Rep. Tokyo Kyoiku Daigaku 6 (1958), nc. 150, 83-142.

[ 7 ] K. Morita, Y. Kawada and H. Tachikawa, On injective modules, Math. Zeit. 68 (1957), 217-226.

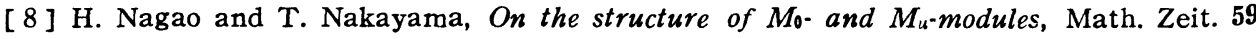
(1953), 164-170.

[9] T. Nakayama, On algebras with complete homology, Abh. Math. Semi. Univ. Hamburg 22 (1958), 300-307.

[10] H. Tachikawa, A characterization of QF-3 algebras, Proc. Amer. Math. Soc. 13 (1962), 701-703.

[11] H. Tachikawa, On dominant dimensions of QF-3 algebras, Trans. Amer. Math. Soc. 112 (1964), 249-266.

[12] R. M. Thrall, Some generalizations of quasi-Frobenius algebras, Trans. Amer. Math. Soc. 64 (1948), 173-183.

University of California, Berkely 